\title{
Cátedra de Residencia Docente de la Carrera de Ciencias de la Educación
}

\author{
Liliana Sanjurjo \\ Alicia Caporossi \\ (Escuela de Ciencias de la Educación) \\ (Universidad Nacional de Rosario. Argentina)
}

La Cátedra de Residencia Docente de la Carrera de Ciencias de la Educación de la Escuela de Ciencias de la Educación y 12 Institutos de Formación Docente del sur de la provincia de Santa Fe han desarrollado el Proyecto de Articulación e Integración aprobado por el Instituto Nacional de Formación Docente durante el año 2009: "La construcción del conocimiento profesional docente en los primeros procesos de socialización profesional”. En el marco de dicho proyecto se organizaron y concretaron:

- La Jornada de Profesores de Práctica el 20 de agosto de 2009 en los salones de la Sede de Gobierno de la UNR. La apertura estuvo a cargo de la Prof. y Mg. Iris Alfonso, en el panel de apertura: "Estado actual del debate acerca de la formación en la práctica profesional docente" coordinado por la Prof. Alicia Caporossi. Estuvieron presentes la Dra. Liliana Sanjurjo, la Prof. y Lic. Ana María Hernández, la Prof. María Fernanda Foresi y la Prof. Susana Gaitán. La Prof. María Fernanda Foresi presentó el sitio virtual de la Red de Prácticas. Los 100 profesores de práctica de esta provincia y de las provincias de Buenos Aires y de Córdoba que participaron en esta jornada trabajaron en grupos experiencias innovadoras o alternativas que toman a la práctica como eje vertebrador de la formación docente y la inserción gradual y progresiva de las prácticas de los estudiantes de los IFD en las escuelas asociadas durante todo el trayecto de la formación inicial. El cierre lo coordinó la Prof. Ana España.

- Los Talleres para Acompañamiento de Docentes Noveles se llevaron a cabo en los meses de septiembre, octubre y noviembre en los Institutos 
de Formación Docente que participan en este Proyecto: $\mathrm{N}^{\circ} 3$ de Villa Constitución, N ${ }^{\circ} 5$ de Cañada de Gómez, N ${ }^{\circ} 7$ de Venado Tuerto, N ${ }^{\circ}$ 24 de Villa Gobernador Gálvez, N ${ }^{\circ} 33$ de Armstrong, N ${ }^{\circ} 37$ de Alcorta y $\mathrm{N}^{\circ} 11, \mathrm{~N}^{\circ} 16, \mathrm{~N}^{\circ} 28, \mathrm{~N}^{\circ} 29, \mathrm{~N}^{\circ} 34, \mathrm{~N}^{\circ} 36$ de la ciudad de Rosario, coordinados por los profesores que integran el Proyecto de Articulación e integración. Estos Talleres se enmarcaron en la necesidad y la conveniencia de que los Institutos de Formación Docente concretaran acciones de seguimiento y apoyo a sus graduados en los primeros procesos de socialización profesional, articulando la formación inicial, el desarrollo profesional y la capacitación.

En el marco del Proyecto de investigación PICTO 2005: "La construcción del conocimiento profesional docente en los primeros procesos de socialización profesional. La inserción de los graduados docentes en la zona sur de la Provincia de Santa Fe" que desarrolla la Cátedra de Residencia Docente de la Carrera de Ciencias de la Educación de la Escuela de Ciencias de la Educación y 14 Institutos de Formación Docente del sur de la provincia de Santa Fe se ha realizado el Seminario "El sofware AQUAD en el análisis computarizado de datos cualitativos". Los profesores investigadores que integran este proyecto en este seminario conocieron los distintos modelos de análisis computarizado de datos cualitativos, las características de AQUAD y desarrollaron un proceso de análisis de datos cualitativos por computadora con dicho programa.

El miércoles 12 de agosto de 2009 integrantes de la Cátedra de Residencia Docente de la Carrera de Ciencias de la Educación de la Escuela de Ciencias de la Educación y docentes de Institutos de Formación Docente, Dra. Liliana Sanjurjo, Lic. y Prof. Ana María Hernández, Mgter. y Prof. Iris Alfonso, Prof. Alicia Caporossi, Prof. María Fernanda Foresi y Prof. Ana España presentaron en el Auditorio "Rodolfo Scholer" de Homo Sapiens Libros de la ciudad de Rosario el libro "Los dispositivos para la formación de las prácticas profesionales", Ediciones Homo Sapiens. El libro es el resultado del trabajo sistemático y sostenido en el tiempo que las autoras desarrollan en el campo de la formación docente.

El 15 y 16 de octubre de 2009 la Escuela de Ciencias de la Educación, Facultad de Humanidades y Artes, UNR organizó las II Jornadas de Formación Docente Universitaria "El porvenir de la Formación Docente Universitaria; entre tensiones y alternativas". Participaron de estas Jornadas destacados profesionales de distintas Universidades y Facultades del país como: Andrea Alliaud, Cristina Alberdi, Ricardo Navarro, Pablo Urbaitel, Susana Marchisio, Myriam Stanley, Marta Brovelli, Elsie Laurino, Celia E. Machado, Jorge Cocca, Luis Garcés, Aldo Lo Russo, Anahí Mastache, 
Gustavo Teres, Norma Valentino, Violeta Castillo, Gustavo Brufman, María Laura Méndez. Además se presentaron los libros: -"Sobre viejos y nuevos saberes. Educación, trabajo y producción en la Provincia de Santa Fe" de Laborde Editor, cuyos autores son Edgardo Ossanna (Coordinador), Mirta Moscatelli, Elisa Welti, María del Carmen Fernández, Alberto Pérez, María Eugenia Guida y Gaspar Tomino. Lo presentaron: Enrique Barés, Luis Garcés y Edgardo Ossanna. -"Los dispositivos para la formación en las prácticas profesionales"-, publicado por Homo Sapiens. Sus autoras: Liliana Sanjurjo (Coordinadora), Alicia Caporossi, Ana España, Ana María Hernández, Iris Alfonso y María Fernanda Foresi.

La Dra. Liliana Sanjurjo, docente titular de la Cátedra de Residencia Docente de la Facultad de Humanidades y Artes de la Universidad Nacional de Rosario, participó como invitada en la Comisión de Cambio Curricular para la Formación Docente de la Provincia de Neuquén en el mes de abril y en el Congreso, llevado a cabo en el mes de octubre en la Universidad Nacional del Comahue que abordó el tema "Investigación educativa y compromiso social" participando con la Prof. Susana Barco y la Dra. Edith Litwin del panel "El oficio de enseña".

La Dra. Liliana Sanjurjo, docente titular de la Cátedra de Residencia Docente de la Facultad de Humanidades y Artes de la Universidad Nacional de Rosario, en forma conjunta con su equipo de trabajo conformado por la Prof. y Lic. Ana María Hernández, la Prof. Alicia Caporossi, la Prof., Lic. y Esp. María Fernanda Foresi, la Mg. Iris Alfonso y la Prof. y Esp. Ana España han participado en el Congreso Nacional e Internacional de Aula Hoy organizado por Homo Sapiens en la ciudad de Rosario, provincia de Santa Fe en la conferencia "Razones que fundamentan nuestra mirada acerca de la formación en las prácticas", los días 10, 11 y 12 de septiembre de 2009.

La cátedra de Residencia, a través de la Profesora titular de la misma, ha participado del homenaje a la Prof. Leonor Bella de Paz, llevado a cabo el 2 de setiembre del corriente año. 